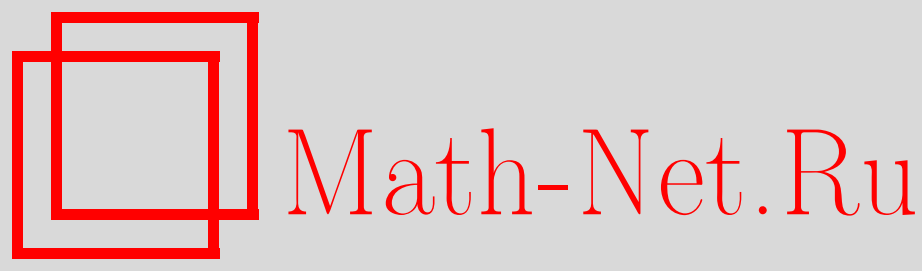

П. П. Физиев, Д. В. Ширков, Решения уравнения Клейна-Гордона на многообразиях с изменяемой геометрией с использованием размерной редукции, ТМФ, 2011, том 167, номер 2, 323-336

DOI: https://doi.org/10.4213/tmf6643

Использование Общероссийского математического портала Math-Net.Ru подразумевает, что вы прочитали и согласны с пользовательским соглашением http://www.mathnet.ru/rus/agreement

Параметры загрузки:

IP: 18.208 .226 .222

26 апреля 2023 г., 14:07:51

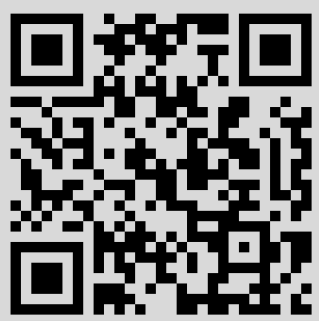




\title{
ФИЗИКА
}

Том 167, № 2

май, 2011

(C) 2011 г. П. П. Физиев*†, Д. В. Ширков ${ }^{\dagger}$

\section{РЕШЕНИЯ УРАВНЕНИЯ КЛЕЙНА-ГОРДОНА НА МНОГООБРАЗИЯХ С ИЗМЕНЯЕМОЙ ГЕОМЕТРИЕЙ С ИСПОЛЬЗОВАНИЕМ РАЗМЕРНОЙ РЕДУКЦИИ}

\begin{abstract}
Развивается недавнее предложение использовать размерную редукцию от четырехмерного пространства-времени $(D=1+3)$ к варианту с меньшим числом пространственных измерений $D=1+d ; d<3$ на достаточно малых расстояниях для создания перенормируемой квантовой теории поля. Изучается уравнение Клейна-Гордона для ряда игрушечных примеров ("обучающих игрушек”) пространства-времени с варьируемой геометрией пространства, включая переход к размерной редукции. Рассмотренные примеры содержат комбинацию двух областей с простой геометрией (двумерные цилиндрические поверхности разного радиуса), соединенных переходной областью. Полезным оказывается новый прием, переводящий решение уравнения Клейна-Гордона на пространствах с переменной геометрией к решению одномерного стационарного уравнения Шредингера с потенциалом, генерируемым изменением геометрии. Сделаны следующие выводы: (1) Сигнал, связанный со степенью свободы, присутствующей лишь в высокоразмерной части многообразия, не проникает в низкоразмерную его часть. Причиной этого является инерциальная сила, неизбежно возникающая в области перехода (в наших моделях это центробежная сила). (2) Специфический спектр скалярных возбуждений напоминает спектр реальных частиц. Он обусловлен геометрией области перехода и представляет ее "отпечатки пальцев". (3) Нарушение пространственной четности, обусловленное асимметричным характером построения наших моделей, может быть связано с нарушением СР-инвариантности.
\end{abstract}

Ключевые слова: размерная редукция, пространства с изменяемой геометрией, уравнение Клейна-Гордона, спектр скалярных возбуждений, нарушение СР-инвариантности.

\section{1. ВВЕДЕНИЕ}

Главная проблема стандартной квантовой гравитации с классическим действием Эйнштейна-Гильберта связана с тем, что постоянная Ньютона $G_{D}$ обладает отрицательной массовой размерностью $\left[G_{D}\right]=\left[M^{2-D}\right]$, где $D=1+d-$ размерность

*Sofia University "St. Kliment Ohridski", Sofia, Bulgaria

† Объединенный институт ядерных исследований, Дубна, Московская обл., Россия. E-mail: fiziev@theor.jinr.ru, shirkovd@theor.jinr.ru 
пространства-времени. Квантовая гравитация не перенормируема по теории возмущений в случае $D>2$. Это справедливо и для электрослабой теории без полей Хиггса. Данное исследование примыкает к работе [1], где было предложено простое паллиативное средство для временной нейтрализации проблемы неперенормируемости. Суть его в том, чтобы применить размерную редукцию (см. также [2]) от многообразия с размерностью $(1+3) \equiv 4$ к многообразию с меньшей размерностью $(1+d) ; d<3$ при достаточно малых расстояниях (больших переданных импульсах).

Наш подход не предполагает модификации понятия времени. Вместо этого мы подразумеваем некоторую достаточно гладкую редукцию пространственных измерений. В результате физическое пространство является непрерывным, но, возможно, не бесконечно гладким многообразием и состоит из частей с различными топологическими размерностями. Ниже будут использованы некоторые игрушечные модели пространства с переменной геометрией. Для наработки физической интуиции и опыта мы начнем со скалярно-волновых решений уравнения Клейна-Гордона (УКГ).

Рассмотрим УКГ для комплексного скалярного поля $\varphi(x)$ на некотором $(1+d)$ мерном пространстве-времени с сигнатурой $\{+,-, \ldots,-\}, \mu, \nu=0,1, \ldots, d, x=$ $\left\{x^{0}, x^{1}, \ldots, x^{d}\right\}=\left\{x^{0}, \mathbf{x}\right\}$, где $x^{0}=t$ и $d \geqslant 1$ - целое число измерений пространства. УКГ записывается следующим образом:

$$
\square \varphi-M^{2} \varphi=0, \quad \square=-\frac{1}{\sqrt{|g|}} \partial_{\mu}\left(\sqrt{|g|} g^{\mu \nu} \partial_{\nu}\right) .
$$

Обычно после квантования [3] (см. также гл. 1 в монографии [4] и книгу [5]) комплексное поле $\varphi(x) \neq \varphi^{*}(x)$ соответствует заряженным частицам с электрическим зарядом $Q= \pm 1$ и (действительной) массой $M$ с током $j_{\mu}=i\left(\varphi^{*} \partial_{\mu} \varphi-\varphi \partial_{\mu} \varphi^{*}\right)$, подчиняющимся уравнению непрерывности, которое дает закон сохранения заряда.

Простейшая возможность для выбора системы отсчета состоит в использовании гауссовых координат, в которых $(1+d)$-мерный оператор $\square$ приобретает (по крайней мере, локально) вид

$$
\square=-\partial_{t t}^{2}+\Delta_{d}, \quad \Delta_{d}=\frac{1}{\sqrt{|\gamma|}} \partial_{m}\left(\sqrt{|\gamma|} \gamma^{m n} \partial_{n}\right), \quad m, n=1, \ldots, d,
$$

с $d$-мерным лапласианом $\Delta_{d}$, определенным $d$-мерной метрикой $\gamma_{m n}$ стандартным образом. Итак, с самого начала мы работаем в рамках $(1+d)$-формализма с глобальным временем, что является обязательным для создания правильной физической картины, особенно для квантовой теории поля. Как следствие, для наших решений частота $\omega$ не будет меняться в частях различных размерностей.

\section{2. УКГ В ЦИЛИНДРИЧЕСКОЙ ПРОСТРАНСТВЕННОЙ ГЕОМЕТРИИ}

\section{1. УКГ в размерности $(1+2)$ с цилиндрической пространственной сим-} метрией. Рассмотрим УКГ

$$
\square \varphi-M^{2} \varphi=-\partial_{t t}^{2} \varphi+\Delta_{2} \varphi-M^{2} \varphi=0
$$

на двумерном многообразии $\mathbb{M}_{\phi z}^{2}$ с цилиндрической симметрией. В декартовых координатах оно определено функцией профиля $\rho(z)$ как поверхность вращения в трехмерном евклидовом многообразии $\mathbb{R}_{X^{1} X^{2} X^{3}}^{3}: X^{1}=\rho(z) \cos \phi, X^{2}=\rho(z) \sin \phi, X^{3}=z$. 
Ограничение на евклидов трехмерный интервал $(d L)^{2}=\left(d X^{1}\right)^{2}+\left(d X^{2}\right)^{2}+\left(d X^{3}\right)^{2}$ на двумерном многообразии $\mathbb{M}_{\phi z}^{2}$ записывается как

$$
(d l)^{2}=\gamma_{m n} d x^{m} d x^{n}=\rho^{2} d \phi^{2}+\left(1+\rho^{\prime 2}\right) d z^{2}, \quad \rho^{\prime}=\frac{d \rho}{d z} .
$$

Лапласиан в явной форме

$$
\Delta_{2}=\frac{1}{\rho^{2}}\left(\partial_{\phi \phi}^{2}+\frac{\rho}{\sqrt{1+\rho^{\prime 2}}} \partial_{z} \frac{\rho}{\sqrt{1+\rho^{\prime 2}}} \partial_{z}\right)
$$

допускает разделение переменных $\varphi(t, \phi, z)=T(t) \Phi(\phi) Z(z)$, дающее систему обыкновенных дифференциальных уравнений (ОДУ). Два из них весьма просты: $T^{\prime \prime}+$ $\omega^{2} T=0 \Rightarrow T(t)=e^{-i \omega t}$ и $\Phi^{\prime \prime}+m^{2} \Phi=0 \Rightarrow \Phi(\phi)=e^{i m \phi}, m=0, \pm 1, \pm 2, \ldots$. Единственное нетривиальное уравнение - уравнение на функцию $Z(z)$

$$
\begin{aligned}
& \frac{1}{\rho \sqrt{1+\rho^{\prime 2}}} \partial_{z}\left(\frac{\rho}{\sqrt{1+\rho^{\prime 2}}} \partial_{z} Z\right)+\left(\omega^{2}-M^{2}-\frac{m^{2}}{\rho^{2}(z)}\right) Z=0 \Rightarrow \\
& \Rightarrow Z(z)=Z(z ; \omega, m) .
\end{aligned}
$$

Оно содержит примечательный член $m^{2} / \rho^{2}(z)$, имеющий вид потенциальной энергии центробежной силы, действующей при $m \neq 0$. Заметим, что этот член представляет простейший пример инерциальной силы с прозрачным физическим смыслом. Подобные силы неизбежны при движении в искривленном пространстве-времени. Они возникают в переходных областях между частями пространств с разными топологическими измерениями. Поэтому появляется надежда кое-что узнать о возможных физических эффектах инерциальных сил, действующих в описанном выше классе искривленных пространств-времен с переменной размерностью.

\section{2. Преобразование задачи к решению ОДУ типа уравнения Шредин-} гера. Полезное математическое свойство для изучения решений уравнения (2.4) состоит в следующем.

ТЕОРема. Уравнение (2.4) может быть преобразовано к ОДУ типа стационарного уравнения Шредингера:

$$
U^{\prime \prime}(u)+(E-V(u)) U(u)=0 .
$$

Следует заметить, что возможность сведения решения УКГ к решению ОДУ типа уравнения Шредингера возникает благодаря разделению переменных в УКГ. Кроме того, используется тот факт, что любая двумерная метрика $\gamma_{m n}$ является конформно плоской. Выполняя преобразование координаты $z \rightarrow u$ и функции профиля $\rho(z) \rightarrow \varrho(u)=\rho(z(u)):$

$$
\begin{gathered}
z \mapsto u: u(z)=\int \sqrt{1+\left(\rho^{\prime}(z)\right)^{2}} \frac{d z}{\rho(z)}, \\
u \mapsto z: z(u)=\int \sqrt{\varrho^{2}(u)-\left(\frac{d \varrho}{d u}\right)^{2}} d u,
\end{gathered}
$$


мы получаем лапласиан $(2.3)$ в виде $\Delta_{2}=\varrho^{-2}\left(\partial_{\phi \phi}^{2}+\partial_{u u}^{2}\right)$, который показывает, что для наших задач функция $\varrho^{2}(u)=\rho^{2}(z(u))$ в точности является двумерным конформным фактором. В переменной $u$ уравнение (2.4) приобретает вид (2.5):

$$
U^{\prime \prime}(u)+\left(-m^{2}-\left(M^{2}-\omega^{2}\right) \varrho^{2}(u)\right) U(u)=0
$$

при отождествлении $E=0, V(u)=\left(M^{2}-\omega^{2}\right) \varrho^{2}(u)+m^{2}, Z(z)=U(u(z))$.

Таким образом, изучение УКГ на рассматриваемых искривленных многообразиях сводимо к решениям уравнения типа Шредингера с потенциалом $V(u)$, определенным геометрией. Обширная литература, посвященная свойствам таких уравнений и их решений для конкретных потенциалов $V(u)$ (см., например, [6], [7]) может быть теперь использована для нашей задачи. Заметим также, что в уравнении (2.7) нет спектрального параметра, подобного обычной квантово-механической энергии $E$ в (2.5), которая складывается с членом $E-V$. Вместо этого специфический спектральный параметр $\omega$ в нашей задаче входит в фактор $M^{2}-\omega^{2}$ в потенциальной функции $V(u)$. Эта деталь своеобразно изменяет хорошо известные спектры квантовых задач, представленных в литературе. В частности, некоторые решения ниже основного состояния (2.5), не имеющие физического смысла в обычном квантово-механическом случае, здесь как бы "всплывают в физическую область" в контексте УКГ благодаря изменению основного состояния, когда $M^{2}-\omega^{2}$ меняет знак.

Этот результат может быть рассмотрен как реализация старой идеи Герца [8] о геометрическом описании физических сил, но теперь на языке волн. Он может быть использован также в теории волноводов в акустике, оптике, для радиоволн и в других областях волной физики.

2.3. Скалярное волновое уравнение в $(1+1)$-мерном пространстве-времени. Для одномерного пространства с координатой $z$ и лапласианом $\Delta_{1}=\partial_{z z}^{2}$ волновая функция имеет простую форму:

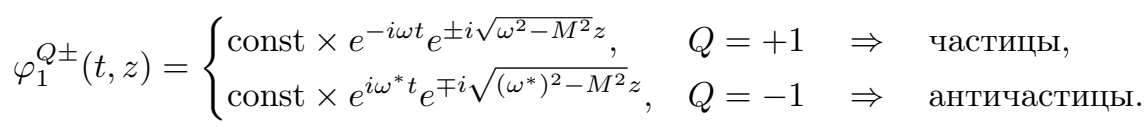

Дополнительный верхний индекс \pm отвечает знаку импульса $p_{z}= \pm k= \pm \sqrt{\omega^{2}-M^{2}}$ или комплексно-сопряженного импульса $p_{z}^{*}= \pm k^{*}= \pm \sqrt{\left(\omega^{*}\right)^{2}-M^{2}}$. При соглашении $\operatorname{Re} \omega \geqslant 0$ в решениях для частиц мы получаем стандартную релятивистскую комбинацию $+i\left(\omega t-p_{z} z\right)=+i p x$, а в решениях для античастиц $-i\left(\omega^{*} t-p_{z}^{*} z\right)=$ $(+i p x)^{*}$.

\section{3. НЕКОТОРЫЕ ЯВНЫЕ ПРИМЕРЫ}

3.1. Два цилиндра радиусов $R$ и $r<R$, соединенные частью конуса. Обратимся к простому примеру двумерного пространства [1]: поверхности двух цилиндров радиусов $R$ и $r<R$ непрерывно связаны частью конуса (см. рис. 1 ). Пусть ось симметрии - это горизонтальная ось $O z$ с началом, расположенным в вершине конуса. Тогда функция профиля $\rho(z)$ (см. рис. 2) имеет вид

$$
\rho(z)= \begin{cases}R=\mathrm{const}, & z \in\left[z_{R},+\infty\right), \\ z \operatorname{tg} \alpha, & z \in\left[z_{r}, z_{R}\right], \\ r=\mathrm{const}, & z \in\left(-\infty, z_{r}\right] .\end{cases}
$$




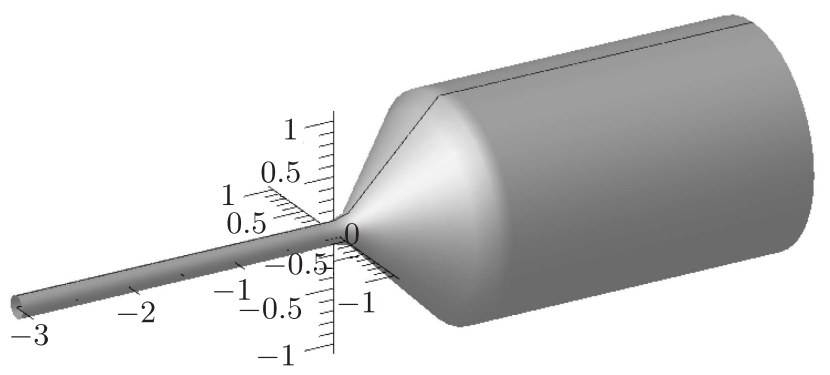

Рис. 1. Два цилиндра радиусов $R$ и $r<R$, соединенные непрерывно частью конуса.

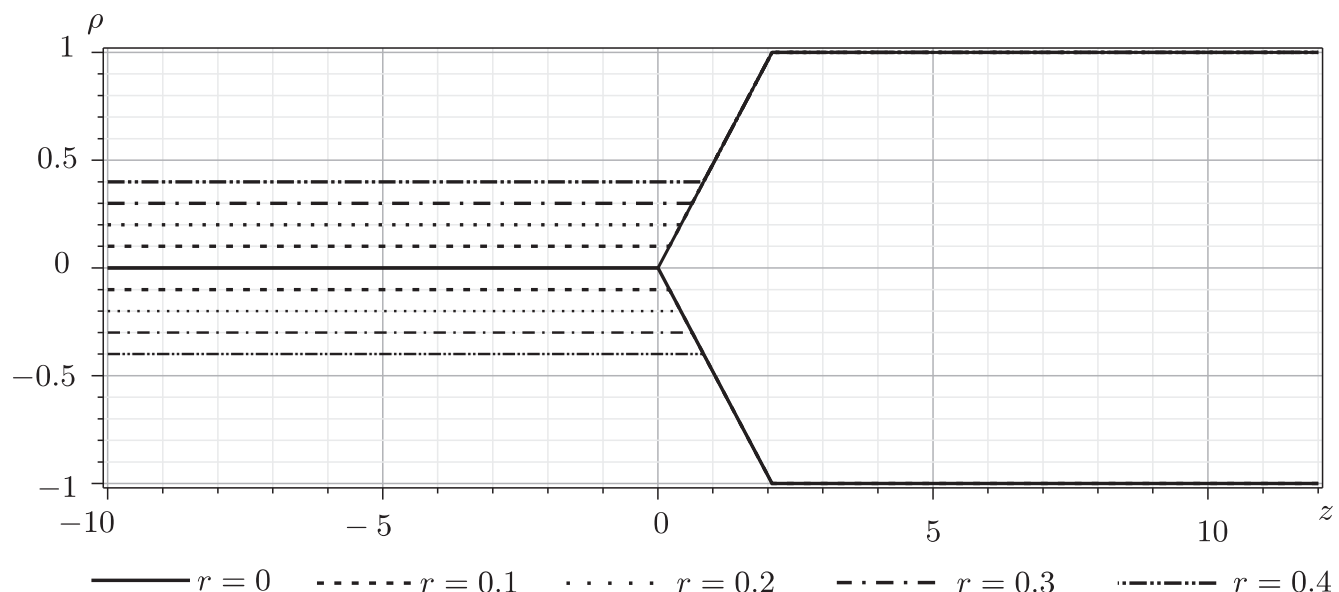

Рис. 2. Сечения непрерывного, но не гладкого многообразия, образованного цилиндрами с радиусами $R=1$ и $r=0,0.1,0.2,0.3,0.4$, связанные частью конуса с углом раствора $\alpha=\pi / 7$.

Здесь $\alpha \in(0, \pi / 2)$ - это половина фиксированного угла при вершине конуса и $z_{R}=$ $R \operatorname{ctg} \alpha>0, z_{r}=r \operatorname{ctg} \alpha>0$. Следующие выражения для функций $z(u)$ и $\varrho(u)$ (см. рис. 3,4$)$ получены из $(2.6)$ :

$$
\begin{aligned}
& z(u ; R, r, \alpha)= \begin{cases}R u, & u \geqslant u_{R}, \\
R u_{R} e^{u \sin \alpha-\cos \alpha}, & u \in\left[u_{r}, u_{R}\right], \\
r\left(u+\frac{\ln (R / r)}{\sin \alpha}\right), & u \leqslant u_{r},\end{cases} \\
& \varrho(u ; R, r, \alpha)= \begin{cases}R, & u \geqslant u_{R}, \\
R e^{u \sin \alpha-\cos \alpha}, & u \in\left[u_{r}, u_{R}\right], \\
r, & u \leqslant u_{r},\end{cases}
\end{aligned}
$$

где $u_{R}=\operatorname{ctg} \alpha$ и $u_{r}=\operatorname{ctg} \alpha-\ln (R / r) / \sin \alpha$. 


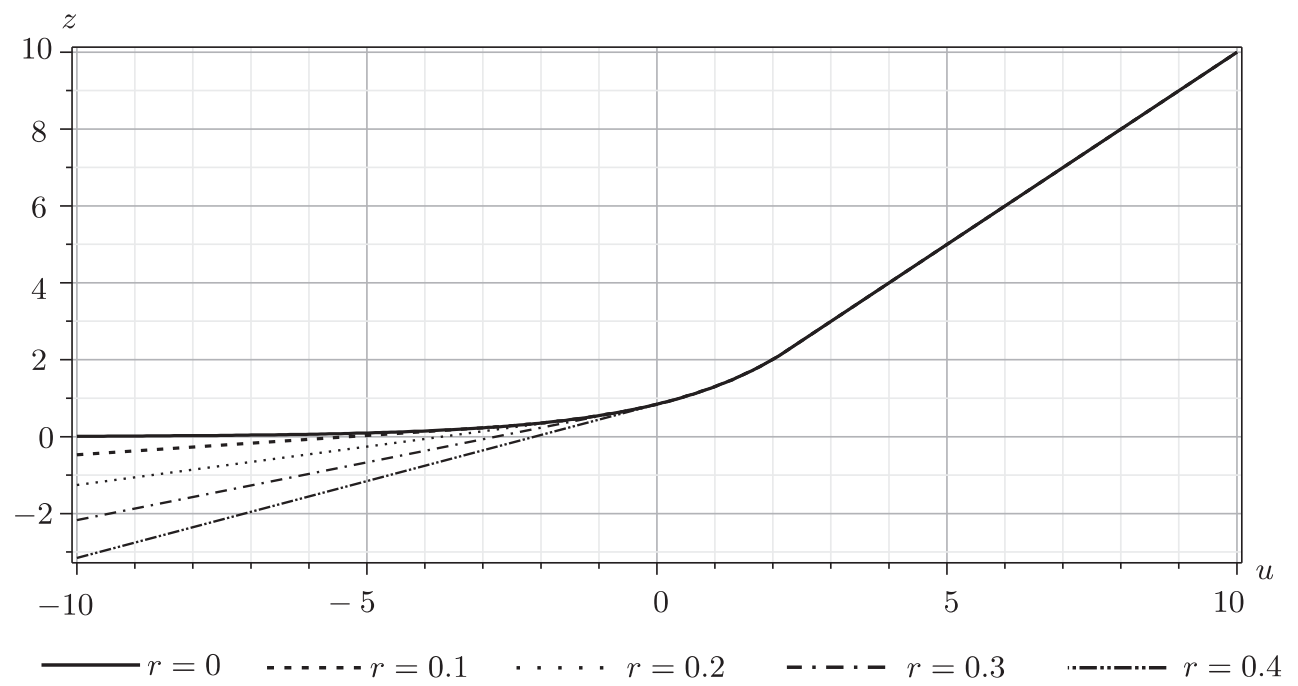

Рис. 3. Предел $r \rightarrow 0$ функции $z(u ; R, r, \alpha)$.

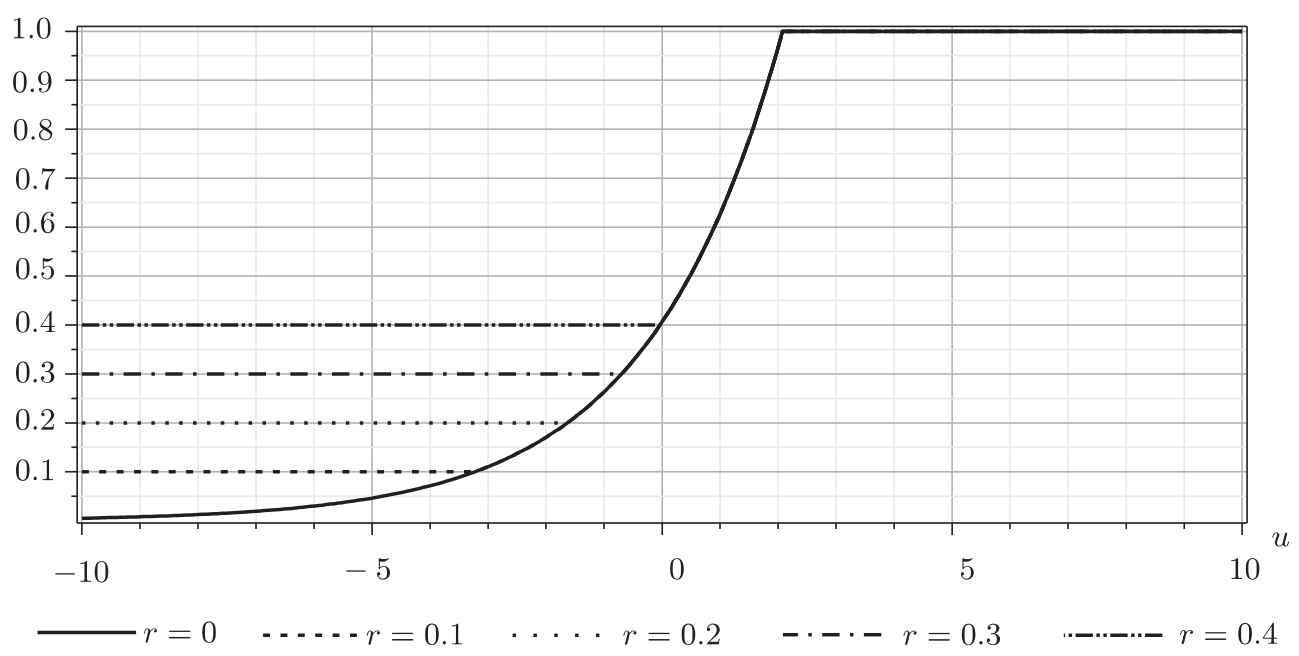

Рис. 4. Предел $r \rightarrow 0$ функции $\varrho(u ; R, r, \alpha)$.

Для волн на поверхности цилиндра с произвольным постоянным радиусом $\rho$ имеет место следующее дисперсионное соотношение:

$$
k_{\rho}=\sqrt{\omega^{2}-M^{2}-\frac{m^{2}}{\rho^{2}}} .
$$

При нормировке: $\varphi_{\omega, m}^{Q \pm}=(a / \sqrt{2}) e^{-i Q \omega t} e^{i m \phi} e^{ \pm k_{\rho} z}$, комплексные решения УКГ с зарядом $Q= \pm 1$ и массой $M$ на цилиндре с радиусом $\rho$ порождают сохраняющийся ток с компонентами

$$
j^{t}=Q|a|^{2} \operatorname{Re} \omega, \quad j^{\phi}=m|a|^{2}, \quad j^{z}= \pm|a|^{2} \operatorname{Re} k_{\rho} .
$$


Как видно, для $m \neq 0$ волна вращается по поверхности цилиндра, так как в (3.5) $J^{\phi} \neq 0$. Это вращение скалярной волны порождает центробежную силу. Становится ясно, что для $m \neq 0$ в пределе $\rho \rightarrow \rho_{\text {crit }}=|m| / \sqrt{\omega^{2}-M^{2}}$ высота барьера центробежного потенциала возрастает неограниченно и останавливает все физические сигналы. В противоположность этому, такое препятствие отсутствует для не вращательного движения вдоль оси $O z$ при $m=0$. Физические сигналы могут распространяться по общей степени свободы двух частей физического пространства с разными топологическими измерениями.

Используя стандартное условие непрерывности функции $Z(z)$ и ее производной $Z^{\prime}(z)$, для волн, идущих направо от $z=-\infty$ к $z=+\infty$ и отражающихся на соединении, получаем (см. обозначения в приложении А)

$$
\begin{aligned}
\vec{Z}(z ; \omega, m ; R, r, \alpha)= & -\Theta_{r}^{+} e^{i k_{r} z_{r}} \times \\
& \times \begin{cases}\frac{2}{\pi} \frac{2 i k_{r} z_{r}}{\Delta} e^{i k_{R}\left(z-z_{R}\right)}, & z \geqslant z_{R}, \\
\frac{2 i k_{r} z_{r}}{\Delta}\left(Y_{R}^{-} J_{\nu}\left(k_{\mathrm{c}} z\right)-J_{R}^{-} Y_{\nu}\left(k_{\mathrm{c}} z\right)\right), & z_{r} \leqslant z \leqslant z_{R}, \\
-e^{i k_{r}\left(z-z_{r}\right)}+\frac{\Delta_{11}}{\Delta} e^{-i k_{r}\left(z-z_{r}\right)}, & z \leqslant z_{r},\end{cases}
\end{aligned}
$$

тогда как для волн, идущих налево от $z=+\infty$ к $z=-\infty$, находим

$$
\begin{aligned}
\overleftarrow{Z}(z ; \omega, m ; R, r, \alpha)=- & \Theta_{R}^{-} e^{-i k_{R} z_{R}} \times \\
& \times \begin{cases}-e^{-i k_{R}\left(z-z_{R}\right)}+\frac{\Delta_{22}}{\Delta} e^{i k_{R}\left(z-z_{R}\right)}, & z \geqslant z_{R}, \\
\frac{2 i k_{R} z_{R}}{\Delta}\left(Y_{r}^{+} J_{\nu}\left(k_{\mathrm{c}} z\right)-J_{r}^{+} Y_{\nu}\left(k_{\mathrm{c}} z\right)\right), & z_{r} \leqslant z \leqslant z_{R}, \\
\frac{2}{\pi} \frac{2 i k_{R} z_{R}}{\Delta} e^{-i k_{r}\left(z-z_{r}\right)}, & z \leqslant z_{r} .\end{cases}
\end{aligned}
$$

Рассмотрим предел $r \rightarrow+0, R=$ const, $\alpha=$ const в соотношении (3.1), когда двумерная поверхность цилиндра с радиусом $r$ и $z \leqslant z_{r}$ превращается в одномерное многообразие - бесконечно тонкую нитку, натянутую вдоль отрицательной части оси $O z$. С геометрической точки зрения это весьма сингулярный предел (см. рис. 3, 4). В этом пределе $z_{R}=R \operatorname{ctg} \alpha$ не изменяется, $z_{r} \rightarrow 0$, а третья часть кусочно-гладких функций $z(u ; R, r, \alpha)(3.2)$ и $\varrho(u ; R, r, \alpha)(3.3)$, заданная на интервале $u \in\left[-\infty, u_{r}\right]$, исчезает, потому что $u_{r} \rightarrow-\infty$. В результате функция формы $\varrho(u ; R, r, \alpha)$ или соответствующий конформный фактор $\varrho^{2}(u ; R, r, \alpha)$ не подходят для описания одномерной части порождающегося непрерывного (но не гладкого) многообразия с изменяющимся измерением, поскольку бесконечный интервал $u \in(-\infty, \infty)$ отображается только на полубесконечный интервал $z \in[0, \infty)$, когда $r=0$.

3.1.1. Состояния непрерывного спектра. Если $J_{R}^{-} \neq 0$, то мы получаем для действительных частот $\omega \geqslant M$

$$
\mathbf{S}(\omega, m ; R, \alpha)=\lim _{r \rightarrow 0} \mathbf{S}(\omega, m ; R, r, \alpha)=-\Theta_{R}^{-} e^{-2 i k_{R} z_{R}} \frac{J_{R}^{+}}{J_{R}^{-}}\left(\begin{array}{ll}
0 & 0 \\
0 & 1
\end{array}\right) .
$$


Очевидны несколько важных следствий, справедливых в случае $m \neq 0$.

1. В пределе $r \rightarrow+0$ коэффициенты перехода $\left|S_{12}\right|^{2}=\left|S_{21}\right|^{2} \equiv 0$. Следовательно, общение посредством волнового сигнала с азимутальным числом $m \neq 0$ между областями с разными измерениями оказывается невозможным.

2. Фактически предел $r \rightarrow+0$ решений, идущих из $z=-\infty$, тривиален для любых $m \neq 0$ :

$$
\vec{Z}(z ; \omega, m ; R, \alpha)=\lim _{r \rightarrow 0} \vec{Z}(z ; \omega, m ; R, r, \alpha) \equiv 0
$$

везде, поскольку фактор $\Theta_{r}^{+}=\Theta\left(\omega-\sqrt{M^{2}+m^{2} / r^{2}}\right) \equiv 0$ для $r<\sqrt{\omega^{2}-M^{2}} /|m|$ в (3.6). Кроме того, $S_{11} \rightarrow 0, S_{12} \rightarrow 0$.

3. В двумерной области пространства для действительных частот $\omega$ модуль коэффициента отражения $S_{22}$ будет $\left|S_{22}\right| \equiv 1$, т.е. происходит полное отражение на конусе от волн, идущих от $z=+\infty$, сопровождающееся изменением фазы рассеянной волны в соответствии с уравнением (3.8). Таким образом, мы получаем следующие нетривиальные решения с $m \neq 0$ :

$$
\begin{aligned}
\overleftarrow{Z}(z ; \omega, m ; R, \alpha) & =\lim _{r \rightarrow 0} \overleftarrow{Z}(z ; \omega, m ; R, r, \alpha)= \\
& =-\Theta_{R}^{-} e^{-i k_{R} z_{R}} \begin{cases}-e^{-i k_{R}\left(z-z_{R}\right)}+\frac{J_{R}^{+}}{J_{R}^{-}} e^{i k_{R}\left(z-z_{R}\right)}, & z \geqslant z_{R}, \\
\frac{2 i k_{R} z_{R}}{J_{R}^{-}} J_{\nu}\left(k_{\mathrm{c}} z\right), & 0 \leqslant z \leqslant z_{R} \\
0, & z \leqslant 0 .\end{cases}
\end{aligned}
$$

Нужно отметить следующие особенности этих решений.

1. Они регулярны и конечны везде, включая сингулярную точку $z=0$, в которой они исчезают.

2. Они не проникают и не распространяются в одномерную часть пространства.

3. Не существует решений с неотрицательными $\omega<\sqrt{M^{2}+m^{2} / R^{2}}$, потому для них фактор $\Theta_{R}^{-}$обращается в нуль. Очевидно, это правильный физический результат, поскольку для $\omega=\sqrt{M^{2}+m^{2} / R^{2}}$ групповая скорость волн исчезает. $\mathrm{K}$ тому же в этом случае длина волн становится бесконечной.

4. Волны, которые распространяются на конусном соединении, обладают комплексным непрерывным спектром импульсов $p_{z}$. Он может быть получен преобразованием Фурье решения (3.9) по переменной $z$. Этот спектр представляет собой своеобразный “отпечаток пальцев", который характеризует геометрию переходной области (в данном случае - конуса). Можно надеяться, изучая этот спектр, восстановить геометрию соединения, по крайней мере, в какой-то степени. Аналогичная задача впервые была поставлена в акустике в 1877 г. Рэлеем, а затем существенно продвинута Вейлем в 1911 г., а позже и многими другими (см. обзорную статью [9] и ссылки в ней).

3.1.2. Резонансные состояния. До настоящего времени мы работали вдали от полюсов матрицы рассеяния $\mathbf{S}$, определенных как нули знаменателя в соотношениях (3.8) или (3.9). Для некоторого $m \neq 0$ рассмотрим дискретную (бесконечную) последовательность частот $\omega_{n, m}, n=0,1, \ldots$, которые подчиняются условию $J_{R}^{-}=0$. Используя безразмерную переменную $\Omega=k_{\mathrm{c}} z_{R}$, запишем спектральное условие в виде $\Omega J_{\nu}^{\prime}(\Omega) / J_{\nu}(\Omega)=\cos \alpha \sqrt{\nu^{2}-\Omega^{2}}$. Типичная картина - отсутствие действительных 


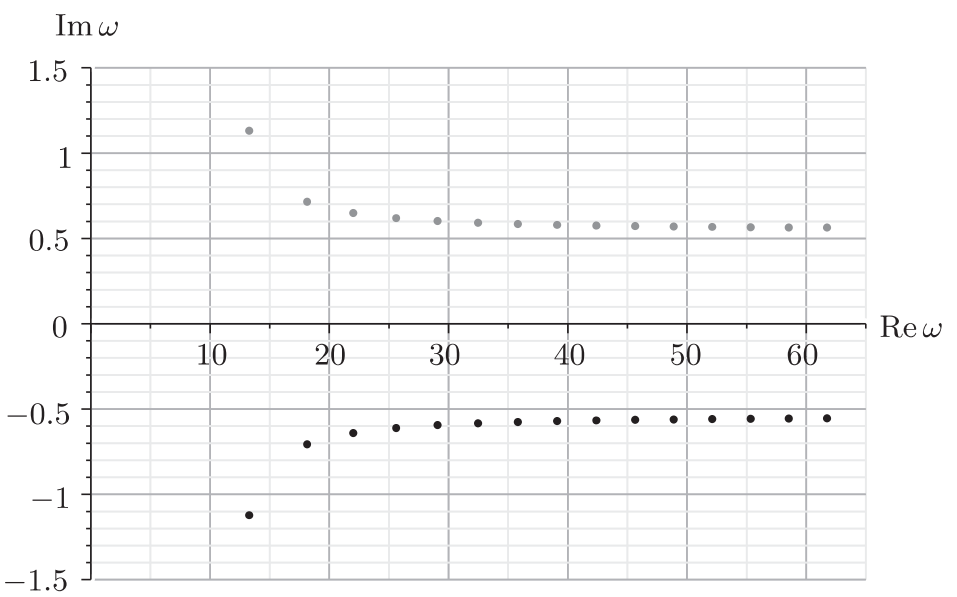

Рис. 5. Комплексный спектр $\omega_{n, m}^{Q}, n=0,1,2, \ldots$, для $m=10, \alpha=\pi / 3$, $M=0$ и $R=\sin \alpha$. Черные точки - частицы, серые точки - античастицы.

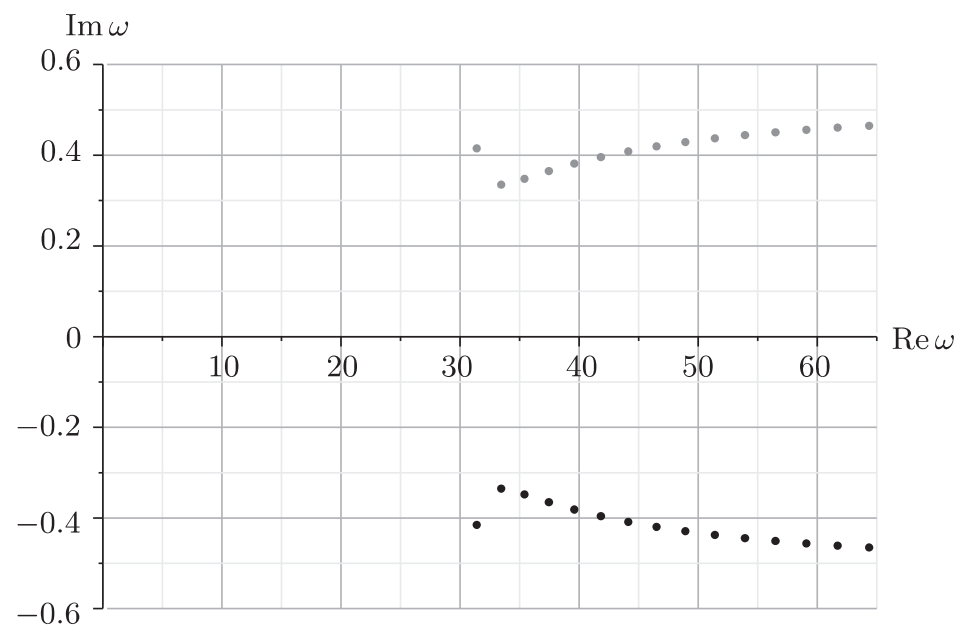

Рис. 6. Комплексный спектр $\omega_{n, m}^{Q}, n=0,1,2, \ldots$, для $m=10, \alpha=\pi / 3$, $M=28.85$ и $R=\sin \alpha$. Черные точки - частицы, серые точки - античастицы.

корней и некоторая последовательность комплексных корней - показана на рис. 5, 6 . Как видно, имеется бесконечное число комплексных резонансных частот

$$
\omega_{n, m}^{Q}=\sqrt{M^{2}+\frac{\sin ^{2} \alpha}{R^{2}}\left(\Omega_{n, m}^{Q}\right)^{2}},
$$

которые соответствуют комплексным корням $\Omega_{n, m}$ и зависят в какой-то степени от массы $M$ скалярного поля (см. рис. 5,6$)$. Спектр $\omega_{n, m}^{Q}$ характеризует геометрию конической формы соединения и непрерывный, но не гладкий переход между двумя цилиндрами. 
Используя асимптотическое разложение функций Бесселя $J_{\nu}(\Omega)$ для больших $|\Omega| \gg \nu=|m| / \sin \alpha$, получаем асимптотики комплексных корней

$$
\begin{gathered}
\Omega_{n, m}^{Q} \approx \pi\left(n+\frac{|m|}{2 \sin \alpha}-\frac{1}{4}\right)-i Q \operatorname{arctg}(\cos \alpha), \\
n=0,1,2, \ldots, \quad m=0, \pm 1, \pm 2, \ldots .
\end{gathered}
$$

Выбор знака мнимой части $\Omega_{n, m}^{Q}$ в $(3.11)$ обеспечивает экспоненциальное затухание полевых возбуждений, когда $t \rightarrow+\infty$, как для частиц $Q=+1$, так и для античастиц $Q=-1$.

Простая формула (3.11) отражает в какой-то степени влияние геометрии соединения от двумерного к одномерному пространству на физику резонансных возбуждений поля Клейна-Гордона.

Когда угол раствора конуса стремится к верхнему пределу: $\alpha \rightarrow \pi / 2$ и $n \gg|m|$, справедливо соотношение

$$
\omega_{n, m}^{Q} \rightarrow \sqrt{M^{2}+\frac{\pi^{2}}{R^{2}}\left(n+\frac{|m|}{2}-\frac{1}{4}\right)^{2}} .
$$

В этом пределе период полураспада резонансных возбуждений $\tau_{n=0, m}=$ $1 /\left|\operatorname{Im}\left(\omega_{n=0, m}^{Q}\right)\right| \rightarrow \infty$ неограниченно возрастает, т.е. резонансные возбуждения сохраняются очень длительное время.

В противоположном случае, когда угол $\alpha \rightarrow 0$, само приближение (3.11) не действительно, поскольку $\left|\Omega_{n, m}^{Q}\right| \sim \pi \nu \rightarrow \infty$ и условие $\left|\Omega_{n, m}^{Q}\right| \gg \nu$ не выполняется в нужной мере, так как $\left|\Omega_{n, m}^{Q}\right| / \nu \sim \pi>1$, однако $\pi \ngtr 1$. Соответствующие более аккуратные оценки могут быть найдены в работе [10].

Таким образом, мы наблюдаем, что изменение топологического измерения пространства действительно дает новый физический эффект. В частности, эффективный спектр масс возмущений (3.10) и их времен жизни зависит от геометрии соединения двумерного и одномерного пространств.

Используя простейшую нормировку, запишем два эквивалентных представления для резонансных релятивистских волновых функций:

$$
Z_{\mathrm{res}}\left(z ; \omega_{n, m}, m ; R, \alpha\right)= \begin{cases}e^{i k_{R}\left(z-z_{R}\right)}, & z \geqslant z_{R} \\ Z_{\mathrm{res}, \mathrm{c}}\left(z ; \omega_{n, m}, m ; R, \alpha\right), & z_{r} \leqslant z \leqslant z_{R} \\ 0, & z \leqslant z_{r}\end{cases}
$$

где

$$
Z_{\mathrm{res}, \mathrm{c}}\left(z ; \omega_{n, m}, m ; R, \alpha\right)=\frac{\pi}{2} Y_{R}^{-} J_{\nu}\left(k_{\mathrm{c}} z\right) \equiv \frac{2 i k_{R} z_{R}}{J_{R}^{+}} J_{\nu}\left(k_{\mathrm{c}} z\right),
$$

получив попутно некое тождество для функций Бесселя и их производных.

3.2. Коническое соединение с гладким переходом в цилиндр. Рассмотрим специальный случай функции ${ }^{1)} \varrho(u)=R e^{p u} /\left(1+e^{p u}\right)$. В переменных $\rho, z$

1) Другие примеры аналитических решений УКГ на двумерных многообразиях с цилиндрической симметрией см. в работе [10]. 


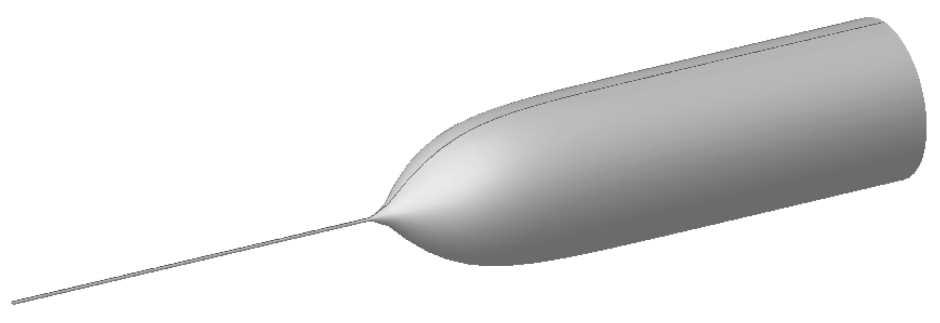

Рис. 7. Коническое соединение с гладким переходом в цилиндр.

соответствующая функция формы поверхности задана уравнением

$$
\frac{z(\rho)}{R}=\frac{1}{p} \ln \frac{1+\sqrt{1-p^{2}(1-\rho / R)^{2}}}{1-\rho / R}-\frac{1}{p} \sqrt{1-p^{2}\left(1-\frac{\rho}{R}\right)^{2}}+\text { const }
$$

определяющим неявным образом двумерную поверхность (см. рис. 7).

Возможные формы ее сечений показаны на рис. 8. В вершине у нас по-прежнему коническая поверхность с углом $\alpha=\operatorname{arctg}\left(p / \sqrt{1-p^{2}}\right)$. Поэтому $p=\sin \alpha$.

При $E=0$ уравнение $(2.5)$ с потенциалом $V(u)=V_{0} /\left(1+e^{-p u}\right)^{2}+m^{2}$, где $V_{0}=R^{2}\left(M^{2}-\omega^{2}\right)$, имеет два независимых решения в виде (см. обозначения в приложении Б)

$$
\begin{aligned}
& U^{ \pm}(u)=A_{ \pm}(u) e^{ \pm m u}= \\
& \quad=\frac{\Gamma\left(c_{ \pm}\right) \Gamma\left(b_{ \pm}-a_{ \pm}\right)}{\Gamma\left(b_{ \pm}\right) \Gamma\left(c_{ \pm}-a_{ \pm}\right)} B_{ \pm}(u) e^{-u \sqrt{V_{0}+m^{2}}}+\frac{\Gamma\left(c_{ \pm}\right) \Gamma\left(a_{ \pm}-b_{ \pm}\right)}{\Gamma\left(a_{ \pm}\right) \Gamma\left(c_{ \pm}-b_{ \pm}\right)} C_{ \pm}(u) e^{+u \sqrt{V_{0}+m^{2}}}
\end{aligned}
$$

При этом $A_{ \pm}(u) \rightarrow 1$ для $u \rightarrow-\infty$, а $B_{ \pm}(u), C_{ \pm}(u) \rightarrow 1$ для $u \rightarrow+\infty$.

Если подставить $|m|$ вместо $\pm m$ на всех местах в формуле (3.14), то из нее получаем решения $\overleftarrow{U}(u ; \omega, m ; R, a)$, являющиеся аналогом $(3.9)$ и также исчезающие на вершине конуса.

Полюсы $S$-матрицы определены уравнениями $b_{+}=-n, n=0,1,2, \ldots$, либо уравнениями $c_{+}-a_{+}=-n, n=0,1,2, \ldots$ В результате приходим к последовательности частот:

$$
\omega_{n, m}=\sqrt{M^{2}+\frac{m^{2}}{R^{2}}-\frac{m^{2}-p^{2} / 4}{R^{2}} W^{2}\left(\frac{(2 n+1) p / 2+|m|}{\sqrt{m^{2}-p^{2} / 4}}\right)}, \quad n=0,1,2, \ldots .
$$

Здесь мы использовали функцию Жуковского $W(x)=(x+1 / x) / 2$. Можно увидеть, что при $M>0$ между частотами (3.15) имеется конечное число вещественных - для $n \in\left[0, n_{\max }\right]$, где $n_{\max }=[\mathcal{N}]$, т. е. равно целой части числа

$$
\mathcal{N}=\sqrt{\left(\frac{M R}{p}\right)^{2}+\left(\frac{m}{p}\right)^{2}}-\frac{|m|}{p}+\sqrt{\left(\frac{M R}{p}\right)^{2}+\left(\frac{1}{2}\right)^{2}}-\frac{1}{2}>0,
$$




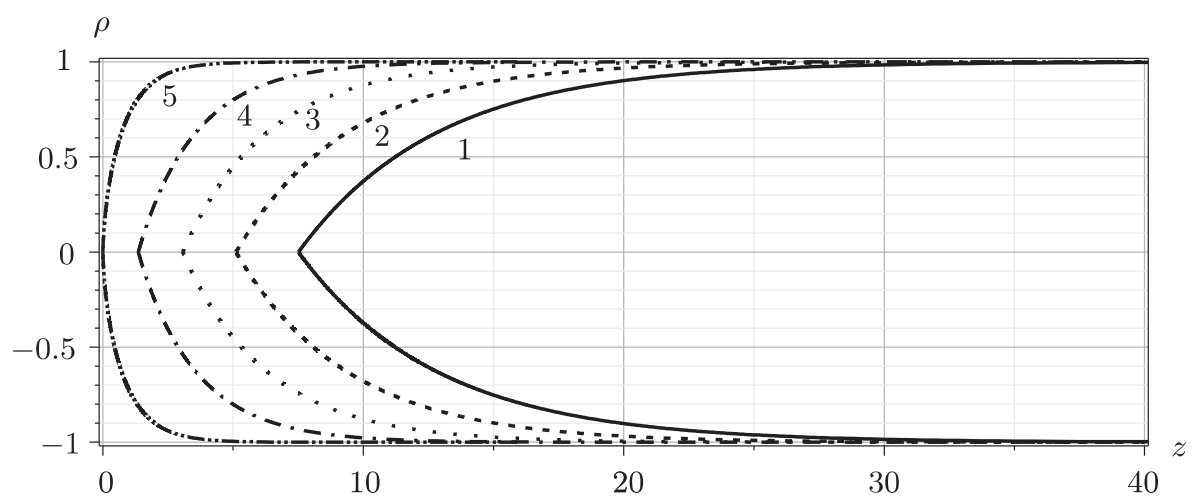

Рис. 8. Части двумерной поверхности $\rho(z)$ для $R=1$ и $p=\sin \alpha=0.185$ (кривая 1), 0.23 (кривая 2), 0.30 (кривая 3), 0.43 (кривая 4), 1.00 (кривая 5).

а также бесконечное число чисто мнимых частот - для $n>n_{\max }$. Так как получаемые из (3.15) вещественные частоты по величине меньше $\sqrt{M^{2}+m^{2} / R^{2}}$, соответствующие им импульсы $\sqrt{\omega_{n, m}^{2}-M^{2}-m^{2} / R^{2}}$ являются чисто мнимыми. Такие решения отвечают сериям связанных состояний с разными $n$ и $m$, которые затухают экспоненциально на пространственной бесконечности.

\section{4. ЗАКЛЮЧЕНИЕ}

Обобщим наши результаты.

1. Доказана полезная теорема, которая соотносит УКГ на пространствах с переменной геометрией уравнению типа Шредингера с эффективным потенциалом, порожденным изменением геометрии. Этот результат основан на разделении переменных в УКГ и на том, что двумерные пространства конформно плоски. Показано, что в случае размерности пространства $d=2$ конформный фактор метрики входит в эффективный потенциал в уравнении типа Шредингера в силу соответствующих замен переменных. Обобщение для размерностей пространства $d>2$ изучается в [11].

2. Как следствие мы заключаем, что получаемые спектры возбуждений полей могли бы служить “отпечатками пальцев" формы области соединения $\rho(z)$. Полученные нетривиальные спектры скалярных возбуждений качественно напоминают некоторые реальные спектры резонансов элементарных частиц.

3. Сигналы, относящиеся к степеням свободы, присущим лишь высокоразмерной части пространства, не распространяются свободно сквозь область соединения (в частности, эти сигналы не проникают в область с низшей размерностью). В нашей игрушечной модели причиной является центробежная сила, действующая на решения с ненулевым угловым моментом. В предельном случае размерной редукции эта сила возрастает до бесконечности и блокирует все решения, кроме решения, имеющего азимутальный номер $m=0$. Можно сказать, что сигналы, которые проникают из высоких измерений в низкие измерения, суть только сигналы, ассоциированные с общими степенями свободы. 
Изучение переменной геометрии в более реалистических $(d=3)$ пространствах представляет несомненный интерес для дальнейшей работы. Этот случай более близок к задачам волновой физики (акустика, волноводы и пр.).

Очевидно нарушение четности вследствие асимметрии пространственной геометрии. Следовательно, пространства с изменяемой геометрией, возможно, дают нам простое основание для описания действительной ситуации с С-, Р- и Т-свойствами частиц.

Второй из перечисленных результатов приводит к новой идее: можно попытаться воспроизвести подобие наблюдаемых спектров элементарных частиц, используя подходящую геометрию области соединения между частями пространства-времени с разными топологическими измерениями.

\section{Краткие обозначения для формул раздела 3}

ПРИЛОЖЕНИЕ А

В задаче с областью конического соединения используются стандартные обозначения для функций Бесселя $J_{\nu}(x)$ и $Y_{\nu}(x)$. Иногда $J_{\nu}(x)$ называют функциями Бесселя, а функции $Y_{\nu}(x)$ - функциями Неймана $\left(N_{\nu}(x) \equiv Y_{\nu}(x)\right)[12],[13]$.

Для волновых чисел волн вдоль оси $z$ на соответствующих цилиндрах приняты обозначения:

$$
k_{R}=\sqrt{\omega^{2}-M^{2}-m^{2} / R^{2}} \quad\left(\operatorname{Re} k_{R} \geqslant 0\right), \quad k_{r}=\sqrt{\omega^{2}-M^{2}-m^{2} / r^{2}} \quad\left(\operatorname{Re} k_{r} \geqslant 0\right) .
$$

На конусе используется величина $k_{\mathrm{c}}=\sqrt{\omega^{2}-M^{2}} / \cos \alpha$. Заметим, что волновое число одномерных волн $m=0$, распространяющихся по оси $O z$ (см. (2.8)), - это просто проекция $k=k_{\mathrm{c}} \cos \alpha=\sqrt{\omega^{2}-M^{2}}(\operatorname{Re} k \geqslant 0)$.

Используются также краткие обозначения:

$$
\begin{gathered}
J_{\nu}^{ \pm}(x, \alpha)=\left(x \frac{d}{d x} \pm i \varkappa_{\nu}(x, \alpha)\right) J_{\nu}(x), \quad Y_{\nu}^{ \pm}(x, \alpha)=\left(x \frac{d}{d x} \pm i \varkappa_{\nu}(x, \alpha)\right) Y_{\nu}(x), \\
\varkappa_{\nu}(x, \alpha)=\cos \alpha \sqrt{x^{2}-\nu^{2}}, \quad \varkappa_{R}=\varkappa_{\nu}\left(k_{\mathrm{c}} z_{R}, \alpha\right)=k_{R} z_{R}, \quad \varkappa_{r}=\varkappa_{\nu}\left(k_{\mathrm{c}} z_{r}, \alpha\right)=k_{r} z_{r}, \\
J_{R}^{ \pm}=J_{\nu}^{ \pm}\left(k_{\mathrm{c}} z_{R}, \alpha\right), \quad J_{r}^{ \pm}=J_{\nu}^{ \pm}\left(k_{\mathrm{c}} z_{r}, \alpha\right), \quad Y_{R}^{ \pm}=Y_{\nu}^{ \pm}\left(k_{\mathrm{c}} z_{R}, \alpha\right), \quad Y_{r}^{ \pm}=Y_{\nu}^{ \pm}\left(k_{\mathrm{c}} z_{r}, \alpha\right), \\
\Delta=J_{R}^{-} Y_{r}^{+}-J_{r}^{+} Y_{R}^{-}, \quad \Delta_{11}=J_{R}^{-} Y_{r}^{-}-J_{r}^{-} Y_{R}^{-}, \quad \Delta_{22}=J_{R}^{+} Y_{r}^{+}-J_{r}^{+} Y_{R}^{+} .
\end{gathered}
$$

Эти обозначения полезны для компактного описания решений и $S$-матрицы в формулах (3.6)-(3.9) и (3.12).

\section{Краткие обозначения в формуле (3.14)}

В формуле (3.14) использованы следующие сокращения:

$$
\begin{aligned}
a_{ \pm} & =\frac{1}{2}+\frac{1}{p}\left(\sqrt{V_{0}+m^{2}}-\sqrt{\frac{p^{2}}{4}+V_{0}} \pm m\right), \\
b_{ \pm} & =\frac{1}{2}+\frac{1}{p}\left(-\sqrt{V_{0}+m^{2}}-\sqrt{\frac{p^{2}}{4}+V_{0}} \pm m\right), \quad c_{ \pm}=1 \pm i \frac{2}{p} m, \\
A_{ \pm}(u) & =\left(1+e^{p u}\right)^{1 / 2-\sqrt{1 / 4+V_{0} / p^{2}}}{ }_{2} F_{1}\left(a_{ \pm}, b_{ \pm} ; c_{ \pm} ;-e^{p u}\right),
\end{aligned}
$$




$$
\begin{aligned}
& B_{ \pm}(u)=\left(1+e^{-p u}\right)^{1 / 2-\sqrt{1 / 4+V_{0} / p^{2}}}{ }_{2} F_{1}\left(a_{ \pm}, a_{ \pm}-c_{ \pm}+1 ; a_{ \pm}-b_{ \pm}+1 ;-e^{-p u}\right), \\
& C_{ \pm}(u)=\left(1+e^{-p u}\right)^{1 / 2-\sqrt{1 / 4+V_{0} / p^{2}}}{ }_{2} F_{1}\left(b_{ \pm}, b_{ \pm}-c_{ \pm}+1 ; b_{ \pm}-a_{ \pm}+1 ;-e^{-p u}\right) .
\end{aligned}
$$

Благодарности. Авторы благодарны И. Арефьевой и О. Теряеву за полезные обсуждения, а Л. Симеонову за помощь в вычислениях. Д. В. Ширков выражает признательность С. Растопчиной за постоянную поддержку на протяжении последних 60 лет. Настоящая работа частично финансировалась Президентской программой поддержки ведущих научных школ (грант 3810.2010.2) и РФФИ (грант № 08-0100686, 11-01-00182), а также Болгарским национальным научным фондом (контракты DO-1-872, DO-1-895 и DO-02-136).

\section{Список литературы}

[1] D. V. Shirkov, Писъма в ЭЧАЯ, 7:6(162) (2010), 625-631, arXiv: 1004.1510.

[2] L. Anchordoqui, De Ch. Dai, M. Fairbairn, G. Landsberg, D. Stojkovic, Vanishing dimensions and planar events at the $L H C$, arXiv: 1003.5914 .

[3] W. Pauli, Rev. Modern Phys., 13:3 (1941), 203-232.

[4] Н. Н. Боголюбов, Д. В. Ширков, Введение в теорию квантованных полей, Наука, М., 1984.

[5] Н.Н.Боголюбов, Д. В. Ширков, Квантовые поля, Наука, М., 1993.

[6] В. де Альфаро, Т. Редже, Потенииальное рассеяние, Мир, М., 1966.

[7] З. Флюгге, Задачи по квантовой механике, т. 1, Мир, М., 1965.

[8] Г. Герц, Принципы механики, изложенные в новой связи, Изд. АН СССР, М., 1959.

[9] W. Arendt, R. Nittka, W. Peter, F. Steiner, "Weyl's Law: Spectral Properties of the Laplacian in Mathematics and Physics", Mathematical Analysis of Evolution, Information, and Complexity, eds. W. Arendt, W. P. Schleich, Wiley-VCH, Weinheim, 2009, 1-71.

[10] P. P. Fiziev, D. V. Shirkov, "Amusing properties of Klein-Gordon solutions on manifolds with variable geometry", The International Workshop "Bogoliubov Readings" (September 22-25, 2010, JINR, Dubna), http://tcpa.uni-sofia.bg/index.php?n=7.

[11] P. P. Fiziev, Riemannian $(\mathbf{1}+\mathbf{d})$-Dim Space-Time Manifolds with Nonstandard Topology which Admit Dimensional Reduction to Any Lower Dimension and Transformation of the Klein-Gordon Equation to the 1-Dim Schrodinger Like Equation, arXiv: 1012.3520.

[12] М. Абрамовиц, И. Стеган (ред.), Справочник по специалъным функциям с формулами, графиками и математическими таблицами, Наука, М., 1979.

[13] И. С. Градштейн, И. М. Рыжик, Таблицы интегралов, сумм, рядов и произведений, Физматлит, М., 1971.

Поступила в редакцию 19.12.2010 